\title{
Streamline Patterns and their Bifurcations near a wall with Navier slip Boundary Conditions
}

Tophøj, Laust; Møller, Søren; Brøns, Morten

Published in:

Physics of Fluids

Link to article, DOI:

$10.1063 / 1.2337660$

Publication date:

2006

Document Version

Publisher's PDF, also known as Version of record

Link back to DTU Orbit

Citation (APA):

Tophøj, L., Møller, S., \& Brøns, M. (2006). Streamline Patterns and their Bifurcations near a wall with Navier slip Boundary Conditions. Physics of Fluids, 18(8), 083102. https://doi.org/10.1063/1.2337660

\section{General rights}

Copyright and moral rights for the publications made accessible in the public portal are retained by the authors and/or other copyright owners and it is a condition of accessing publications that users recognise and abide by the legal requirements associated with these rights.

- Users may download and print one copy of any publication from the public portal for the purpose of private study or research.

- You may not further distribute the material or use it for any profit-making activity or commercial gain

- You may freely distribute the URL identifying the publication in the public portal 


\title{
Streamline patterns and their bifurcations near a wall with Navier slip boundary conditions
}

\author{
Laust Tophøj, Søren Møller, and Morten Brøns ${ }^{a)}$ \\ Department of Mathematics, Technical University of Denmark, Matematiktorvet, \\ Building 303, DK-2800 Kongens Lyngby, Denmark
}

(Received 19 October 2005; accepted 25 July 2006; published online 29 August 2006)

\begin{abstract}
We consider the two-dimensional topology of streamlines near a surface where the Navier slip boundary condition applies. Using transformations to bring the streamfunction in a simple normal form, we obtain bifurcation diagrams of streamline patterns under variation of one or two external parameters. Topologically, these are identical with the ones previously found for no-slip surfaces. We use the theory to analyze the Stokes flow inside a circle, and show how it can be used to predict new bifurcation phenomena. (C) 2006 American Institute of Physics. [DOI: 10.1063/1.2337660]
\end{abstract}

\section{INTRODUCTION}

The no-slip boundary condition at an interface between a viscous fluid and a solid surface has been successfully applied to a large range of flow problems. However, it cannot be deduced from first principles, and has been continuously debated in the scientific literature. Almost 200 years ago Navier proposed a more general boundary condition that allows slip at the surface. The boundary condition states that the tangential component of the velocity $v_{\|}$at the surface is proportional to the rate of strain at the surface, or equivalently, $v_{\|}$is proportional to the tangential stress $\sigma_{\|}$at the surface,

$$
v_{\|}=\frac{\lambda_{0}}{\mu} \sigma_{\|}
$$

Here $\mu$ is the viscosity of the fluid and $\lambda_{0}$, the slip length, describes the "slipperiness" of the surface. In the limit $\lambda_{0}$ $\rightarrow 0$ the standard no-slip boundary condition is recovered, and for $\lambda_{0} \rightarrow \infty$ the fluid flows freely on the surface. The slip length $\lambda_{0}$ has a simple physical interpretation: ${ }^{1}$ If the flow were linearly extended below the surface, a no-slip condition would be satisfied at the depth $\lambda_{0}$.

There is a strong current interest in slip flows in microfluidics. Slip lengths in the $\mathrm{nm}$ to $\mu \mathrm{m}$ range have recently been measured; see, e.g., Refs. 1-5. On the theoretical side it has recently been proved that the slip boundary condition may result from a modeling of rough surfaces. It can be shown analytically ${ }^{6}$ that no-slip Stokes flow over a wall with microscopic grooves on the macroscopic level fulfills a Navier slip boundary condition.

In the present paper we are concerned with the topology of streamlines of the two-dimensional (2D) flow near a surface where the Navier slip condition is satisfied, and, in particular, with changes (or bifurcations) in the streamline patterns as external parameters are varied. A systematic study of bifurcations of streamline topology close to a no-slip wall based on Taylor expansion of the velocity field was per-

\footnotetext{
${ }^{a)}$ Author to whom correspondence should be addressed. Telephone: +45 4525 3067. Electronic mail: m.brons@ mat.dtu.dk
}

formed by Bakker ${ }^{7}$ and extended by Hartnack, ${ }^{8}$ who obtained a simplified expression for the streamfunction, a normal form, by the application of linear and nonlinear coordinate transformations. See also the recent review by Brøns. ${ }^{9}$ We also will apply a normal form approach, but the analysis is different due to the more complicated conditions imposed on the Taylor expansion coefficients by the boundary condition (1).

Flow topology is of particular interest in connection with stirring and mixing. A simple, much studied configuration is Stokes flow inside a circle, driven by rotlets or rollers. ${ }^{10-14}$ Usually, no-slip boundary conditions are considered, but a flow with slip boundary conditions was studied analytically by Palaniappan and Daripa, ${ }^{15}$ and a rich set of flow topologies was obtained. We revisit their work, correct an error, and show how bifurcation theory can be used to find new bifurcation phenomena.

\section{THE NAVIER BOUNDARY CONDITION}

The tangential stress at the surface can be found from the viscous stress tensor

$$
\sigma_{i j}=\mu\left(\partial_{j} v_{i}+\partial_{i} v_{j}\right) .
$$

Letting $\left(t_{i}\right)$ denote a unit vector tangential to the interface and $\left(n_{i}\right)$ a unit normal vector pointing into the fluid, the Navier boundary condition (1) can be expressed as

$$
v_{i} t_{i}=\lambda_{0} t_{i} n_{j}\left(\partial_{j} v_{i}+\partial_{i} v_{j}\right) \text {. }
$$

For a flat wall, the boundary condition reduces to

$$
v_{\|}=\lambda_{0} \partial_{n} v_{\|},
$$

where $v_{\|}$is the tangential velocity, and $\partial_{n} v_{\|}$is its normal derivative.

For a curved wall with radius of curvature $R$, Eq. (3) can be written as ${ }^{16}$

$$
v_{\|}=\left(\frac{1}{\lambda_{0}}-\frac{1}{R}\right)^{-1} \partial_{n} v_{\|}=\lambda_{a} \partial_{n} v_{\|} .
$$

The radius of curvature is counted with sign, $R$ being positive at points where the flow domain is locally convex. The 
effective slip length $\lambda_{a}$ depends on both physical and geometric properties of the flow, and may, in principle, become negative for sufficiently small positive curvatures $R$. This is not relevant if the slip length is microscopic and the curvature macroscopic, but we note that the bifurcation analysis of the present paper is valid for all $\lambda_{a} \neq 0$.

As we consider flow near an impermeable wall, the noflux boundary condition

$$
v_{i} n_{i}=0
$$

will also be imposed.

The subsequent topological analysis is local and is valid in some neighborhood of a point on the surface. We introduce an $X Y$ coordinate system such that the given point is the origin and the surface curve is represented by the graph $Y=f(X)$ with $f(0)=f^{\prime}(0)=0$, that is, the surface is tangent to the $X$ axis at the origin. The flow domain is $Y>f(X)$. The analysis will be simplified by changing coordinates such that the surface curve coincides with a coordinate axis. To this end we consider a map $T$ defined by

$$
\left(\begin{array}{l}
X \\
Y
\end{array}\right)=T\left(\begin{array}{l}
x \\
y
\end{array}\right)=\left(\begin{array}{c}
x-\frac{f^{\prime}(x) y}{\sqrt{1+f^{\prime}(x)^{2}}} \\
f(x)+\frac{y}{\sqrt{1+f^{\prime}(x)^{2}}}
\end{array}\right) .
$$

The Jacobian of $T$ is the identity at the origin, and it follows from the Inverse Function Theorem that $T$ is invertible in a neighborhood of the origin and hence defines a local change of coordinates. The inverse transformation $T^{-1}$ maps the surface curve $Y=f(X)$ to $y=0$. Furthermore, $T^{-1}$ maps lines orthogonal to the surface isometrically to lines orthogonal to the $x$ axis. It follows that

$$
\partial_{n}=\partial_{y} .
$$

We assume the fluid is incompressible and hence a streamfunction $\psi$ exists. One finds that

$$
v_{\|}=\partial_{n} \psi
$$

in the $X Y$ coordinate system, and, according to (8), the boundary condition (5) becomes

$$
\partial_{y} \psi(x, 0)=\lambda_{a}(x) \partial_{y}^{2} \psi(x, 0),
$$

where

$$
\lambda_{a}(x)=\left(\frac{1}{\lambda_{0}}-\frac{1}{R(x)}\right)^{-1} .
$$

The subsequent analysis will hold for any nonzero, sufficiently differentiable function $\lambda_{a}(x)$ in (10). In particular, the results are also valid if the material properties vary along the surface such that $\lambda_{0}=\lambda_{0}(x)$. Clearly, the case of $\lambda_{a}(x)$ being constant is the most basic one, as it includes a planar surface $(1 / R=0)$ and a circular surface with constant radius of curvature $R$. However, a general $\lambda_{a}(x)$ does not complicate the analysis substantially, and, as we shall see, the specific form of $\lambda_{a}(x)$ plays no role for the type of flow topologies and bifurcations that can occur.
The no-flux boundary condition (6) becomes, in terms of $\psi$,

$\psi(x, 0)=0$.

\section{BIFURCATION OF STREAMLINE PATTERNS}

\section{A. Simple and degenerate critical points}

The analysis of streamline patterns will be based on the streamfunction in the $x y$ coordinate system. The streamlines are level curves of $\psi$ and can be found from the differential equations

$$
\dot{x}=\partial_{y} \psi, \quad \dot{y}=-\partial_{x} \psi .
$$

We expand $\psi$ in a Taylor series based at the origin,

$$
\psi(x, y)=\sum_{i, j=0}^{\infty} a_{i j} x^{i} y^{j},
$$

where

$$
a_{i j}=\frac{1}{i ! j !} \partial_{x}^{i} \partial_{y}^{j} \psi(0,0)
$$

Inserting the expansion (14) in the no-flux boundary condition (12) yields

$$
a_{i 0}=0, \quad \text { for } i=0,1, \ldots .
$$

The slip condition (10) also gives a sequence of relations between the Taylor coefficients $a_{i j}$. The first few are

$$
\begin{aligned}
& a_{01}=2 \lambda_{a}(0) a_{02}, \\
& a_{11}=2 \lambda_{a}(0) a_{12}+2 \lambda_{a}^{\prime}(0) a_{02}, \\
& a_{21}=2 \lambda_{a}(0) a_{22}+2 \lambda_{a}^{\prime}(0) a_{12}+\lambda_{a}^{\prime \prime}(0) a_{02} .
\end{aligned}
$$

If $a_{01}=0$, the origin is a critical point for $\psi$, that is, a stagnation point. From the Navier boundary condition (17a), it follows that $a_{02}=0$. With the nondegeneracy condition $a_{11} \neq 0$ the linearization of (13) becomes

$$
\left(\begin{array}{l}
\dot{x} \\
\dot{y}
\end{array}\right)=\left(\begin{array}{cc}
a_{11} & 0 \\
0 & -a_{11}
\end{array}\right)\left(\begin{array}{l}
x \\
y
\end{array}\right) \text {, }
$$

which has a regular saddle point at the origin.

It is a basic result from nonlinear dynamics ${ }^{17}$ that the local behavior of the streamlines in this case is determined by the linearization. Because of the diagonal form of the Jacobian matrix in (18), the dividing streamline is orthogonal to the surface. This is in contrast to flows near a no-slip boundary, where the angle depends on the stress and pressure gradients, ${ }^{8}$

$$
\tan \theta=-3 \frac{\partial \sigma_{\|} / \partial x}{\partial p / \partial x} .
$$

If $a_{11}<0$ the surface curve is tangent to the stable manifold of the stagnation point, which is then a point of separation. If $a_{11}>0$ the surface curve is tangent to the unstable manifold of the stagnation point, which is then a point of attachment. See Fig. 1. 
(a)

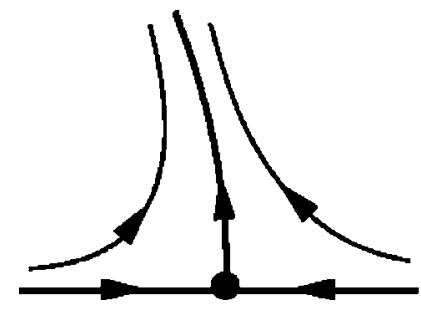

(b)

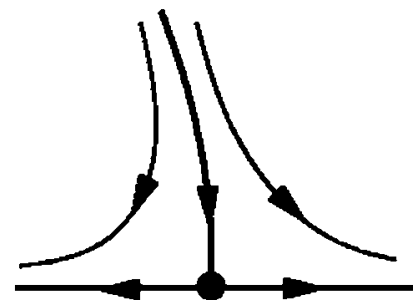

FIG. 1. Local streamline pattern near a regular stagnation point on the surface. The dividing streamline intersects the surface at a right angle. (a) Separation, $a_{11}<0$. (b) Attachment, $a_{11}>0$. Here and in Figs. 2-4, the horizontal line represents the wall $y=0$ in the transformed coordinates defined in (7).

If, further, $a_{11}=0$ the stagnation point is degenerate, and the linearized system (18) does not determine the local structure of the streamlines. Quadratic terms in the streamline equations (13) - the cubic terms in $\psi$-must be taken into account. With the boundary conditions (17a) and (17b) we find

$$
\psi=y\left(a_{21} x^{2}+a_{03} y^{2}\right)+\mathcal{O}_{4},
$$

where $\mathcal{O}_{4}$ denotes terms of leading order 4 in $(x, y)$.

Possible dividing streamlines from the stagnation point are found by solving $\psi(x, y)=0$. In addition to the surface, $y=0$, there are, assuming the nondegeneracy conditions

$$
a_{03} \neq 0, \quad a_{21} \neq 0,
$$

the solutions

$$
y=\sqrt{-\frac{2 a_{21}}{a_{03}}}|x|+\mathcal{O}\left(x^{3 / 2}\right) .
$$

If $a_{03}$ and $a_{21}$ have opposite signs, this represents two dividing streamlines going into the fluid. The two dividing streamlines are symmetric with respect to the surface normal. In the no-slip case, there is, in general, no such symmetry. ${ }^{8}$ If $a_{03}$ and $a_{21}$ have the same sign, there are no dividing streamlines. The two situations are illustrated in Figs. 2(a) and 2(b). We denote this a degeneracy of order 3.

If one of the nondegeneracy conditions in (21) is violated, (22) does not resolve the structure of the flow close to

(a)

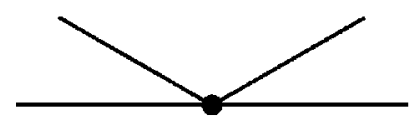

(c)

(b)
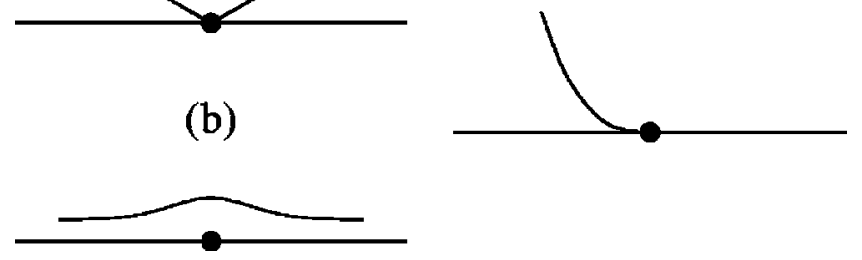

FIG. 2. Local streamline patterns near degenerate critical points on the surface. (a) Here $a_{11}=0, a_{03}$, and $a_{21}$ opposite sign. Two separatrices go into the fluid from the critical point. (b) Here $a_{11}=0, a_{03}$, and $a_{21}$ same sign. No separatrices exist. (c) Here $a_{11}=a_{21}=0, a_{03} \neq 0, a_{31} \neq 0$. A single separatrix, tangent to the surface, enters the flow. the origin. Considering the degeneracy of order 4 , where $a_{21}=0$ but $a_{03} \neq 0$, we have

$$
\psi=y\left(a_{03} y^{2}+a_{31} x^{3}+a_{13} x y^{2}+a_{04} y^{3}\right)+\mathcal{O}_{5} .
$$

Some terms can be removed by the near-identity quadratic transformation

$$
y \mapsto y-\frac{a_{13}}{3 a_{03}} x y-\frac{a_{04}}{3 a_{03}} y^{2},
$$

which yields the normal form

$$
\psi=y\left(a_{03} y^{2}+a_{31} x^{3}\right)+\mathcal{O}_{5} .
$$

If $a_{31} \neq 0$, we find a solution to $\psi=0$ of the form

$$
y=\sqrt{-\frac{a_{31}}{a_{03}} x^{3}}+\mathcal{O}\left(x^{2}\right),
$$

which is defined when $x$ has the opposite sign of $a_{31} / a_{03}$. It represents a single separatrix, tangent to the surface. See Fig. 2(c).

\section{B. Unfolding the degeneracy of order 3}

To analyze how the streamline pattern changes when the parameters take values close to the degeneracy $a_{01}=a_{11}=0$, we consider them as small parameters,

$$
\varepsilon_{1}=a_{01}, \quad \varepsilon_{2}=a_{11},
$$

and impose the nondegeneracy conditions (21). This twoparameter family of streamfunctions is denoted an unfolding of the degeneracy. We aim at obtaining a simplified normal form by coordinate transformations and possibly reduce the number of parameters in the process, thus generating a simpler unfolding. The number of small parameters in the normal form is the codimension of the degeneracy.

As the flow topology of the degenerate case $\varepsilon_{1}=\varepsilon_{2}=0$ depend only on cubic terms of the streamfunction, cf. (20), one would expect that operating on a truncation of the expansion of the streamfunction (14) to third order would suffice. This is indeed the case with no-slip boundary conditions, ${ }^{8}$ but for finite slip lengths the links (17) between expansion coefficients at different orders complicate matters, and errors occur if truncation is done too early in the process. Hence, we will transform the full expansion (14), and only truncate after the coordinate transformations.

To obtain a normal form, we apply a coordinate transformation,

$$
x=x_{0}+A \xi+B \eta, \quad y=\eta, \quad A \neq 0 .
$$

The transformation is chosen to map the surface curve $y=0$ to the line $\eta=0$. Inserting the transformation in $\psi$ and making a Taylor expansion at the origin $(\xi, \eta)=(0,0)$, we obtain an expression of the form

$$
\tilde{\psi}=\eta \sum_{i, j=0}^{\infty} \tilde{a}_{i j+1} \xi^{i} \eta^{j},
$$

where the lowest order coefficients are

$$
\tilde{a}_{01}=\partial_{y} \psi\left(x_{0}, 0\right),
$$




$$
\begin{aligned}
\tilde{a}_{02}= & B \partial_{x} \partial_{y} \psi\left(x_{0}, 0\right)+\frac{1}{2 \lambda_{a}\left(x_{0}\right)} \partial_{y} \psi\left(x_{0}, 0\right), \\
\tilde{a}_{11}= & A \partial_{x} \partial_{y} \psi\left(x_{0}, 0\right), \\
\tilde{a}_{12}= & A B \partial_{x}^{2} \partial_{y} \psi\left(x_{0}, 0\right)+A \frac{\partial_{x} \partial_{y} \psi\left(x_{0}, 0\right)}{2 \lambda_{a}\left(x_{0}\right)} \\
& -A \frac{\lambda_{a}^{\prime}\left(x_{0}\right) \partial_{y} \psi\left(x_{0}, 0\right)}{\lambda_{a}\left(x_{0}\right)^{2}}
\end{aligned}
$$

We can obtain the simplification

$$
\tilde{a}_{11}=0, \quad \tilde{a}_{12}=0,
$$

if

$$
\begin{aligned}
& \partial_{x} \partial_{y} \psi\left(x_{0}, 0\right)=0, \\
& B=\frac{\lambda_{a}^{\prime}\left(x_{0}\right)}{\lambda_{a}^{2}\left(x_{0}\right)} \frac{\partial_{y} \psi\left(x_{0}, 0\right)}{\partial_{x}^{2} \partial_{y} \psi\left(x_{0}, 0\right)} .
\end{aligned}
$$

To solve (32a) for $x_{0}$, we use (14) to obtain

$$
\partial_{x} \partial_{y} \psi\left(x_{0}, 0\right)=\varepsilon_{2}+2 a_{21} x_{0}+\sum_{i=3}^{\infty} i a_{i 1} x_{0}^{i-1}=g\left(x_{0}, \varepsilon_{2}\right) .
$$

From this,

$$
g(0,0)=0, \quad \frac{\partial g}{\partial x_{0}}(0,0)=2 a_{21} \neq 0,
$$

and it follows from the implicit function theorem that there exists a function $x_{0}\left(\varepsilon_{2}\right)$ with $x_{0}(0)=0$ such that $\partial_{x} \partial_{y} \psi\left(x_{0}\left(\varepsilon_{2}\right), 0\right)=0$. By implicit differentiation, one obtains

$$
x_{0}=-\frac{\varepsilon_{2}}{2 a_{21}}+\mathcal{O}\left(\varepsilon_{2}^{2}\right),
$$

and (32b) becomes

$$
B=\frac{\lambda_{a}^{\prime}(0)}{4 a_{21} \lambda_{a}(0)^{2}} \varepsilon_{1}+\mathcal{O}\left(\varepsilon_{1}, \varepsilon_{2}\right) .
$$

With these choices, the transformed streamfunction is

$$
\tilde{\psi}=\eta\left(\tilde{a}_{01}+\frac{\tilde{a}_{01}}{2 \lambda_{a}\left(x_{0}\right)} \eta+\tilde{a}_{21} \xi^{2}+\tilde{a}_{03} \eta^{2}\right)+\mathcal{O}_{4},
$$

where

$$
\begin{aligned}
& \tilde{a}_{01}=\mathcal{O}\left(\varepsilon_{1}, \varepsilon_{2}\right), \\
& \tilde{a}_{21}=A^{2} a_{21}+\mathcal{O}\left(\varepsilon_{1}, \varepsilon_{2}\right), \\
& \tilde{a}_{03}=a_{03}+\mathcal{O}\left(\varepsilon_{1}, \varepsilon_{2}\right) .
\end{aligned}
$$
value,

We can choose $A$ to give $\tilde{a}_{21}$ and $\tilde{a}_{03}$ the same absolute

$$
A=\sqrt{\left|\frac{a_{03}}{a_{21}}\right|}+\mathcal{O}\left(\varepsilon_{1}, \varepsilon_{2}\right),
$$

and divide the streamfunction by a factor $\widetilde{a}_{03}$ to obtain

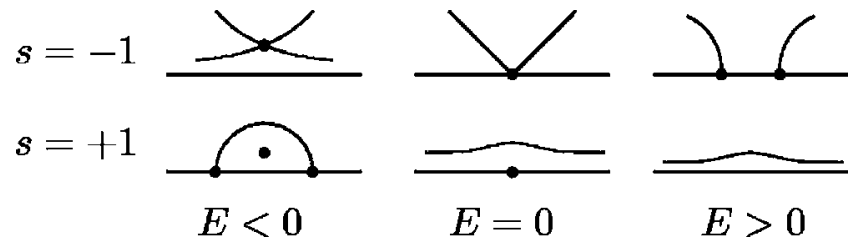

FIG. 3. Bifurcation of streamline patterns for the codimension one normal form (41) as the bifurcation parameter $E$ changes sign. The top row is a bubble merging bifurcation $(s=-1)$, where two critical points on the surface merge and lift off the surface as $E$ is decreased. The bottom row is a bubble creation bifurcation $(s=+1)$; here a recirculating zone attached to the surface is created as $E$ is decreased.

$$
\left.\widetilde{\psi}=\eta(E+\alpha E \eta)+s \xi^{2}+\eta^{2}\right)+\mathcal{O}_{4},
$$

where $E=\tilde{a}_{01} / \tilde{a}_{03}=\mathcal{O}\left(\varepsilon_{1}, \varepsilon_{2}\right)$ is a small parameter, $\alpha=1 /\left(2 \lambda_{a}\left(x_{0}\right) \tilde{a}_{03}\right), \quad$ and $\quad s=\operatorname{sign}\left(\tilde{a}_{21} / \tilde{a}_{03}\right)=\operatorname{sign}\left(a_{21} / a_{03}\right)$. Finally, dropping the $\mathcal{O}_{4}$ term and renaming the coordinates back to $x, y$ we obtain the normal form

$$
\psi=y\left(E+\alpha E y+s x^{2}+y^{2}\right) .
$$

The normal form has only one small parameter, and hence the degeneracy has codimension one.

The analysis of the streamline topology of (41) is straightforward. Critical points on the surface are determined from $\partial_{y} \psi(x, 0)=E+s x^{2}=0$. Thus there are two critical points for $E s<0$ which merge for $E=0$ and disappear for $E s>0$. Critical points off the surface satisfy $\partial_{x} \psi=0$ which gives $x=0$. Then $u(0, y)=3 y^{2}+E=0$ has one positive solution for $E<0$ and no solutions for $E>0$. The type of the critical points is found from the determinant of the Jacobian matrix. If it is positive, the critical point is a center, if it is negative the critical point is a saddle.

The bifurcation diagrams are shown in Fig. 3. For $s=+1$ a recirculating bubble attached to the surface is created or destroyed, and we denote it a (bubble) creation bifurcation. For $s=-1$ the two saddle points on the surface for $E>0$ will typically be ends of recirculating bubbles, attached to the surface away from the local region in other saddle points. Hence, this bifurcation is denoted a (bubble) merging bifurcation.

Note, that the variation of $\lambda_{a}$ along the wall is effectively transformed away in the normal form (41). Hence, both the bubble creation and bubble merging bifurcation may occur in slip flows close to surfaces of arbitrary shape. In other words, the wall shape does not impose any topological constraints on the streamline pattern, although the specific details of the shape of the streamlines will of course depend on $\lambda_{a}(x)$.

\section{Unfolding the degeneracy of order 4}

If one of the nondegeneracy conditions (21) are broken, the analysis above does not hold. Here we consider the case where $a_{21}$ is also a small parameter and hence set

$$
\bar{\varepsilon}=\left(\varepsilon_{1}, \varepsilon_{2}, \varepsilon_{3}\right)=\left(a_{01}, a_{11}, a_{21}\right),
$$

and assume the nondegeneracy conditions

$$
a_{03} \neq 0, \quad a_{31} \neq 0 .
$$


We proceed as in Sec. III B to obtain a normal form, now including terms up to order four in $\psi$. We apply a transformation of the form

$$
x=x_{0}+A \xi+B \eta, \quad y=\eta+C \xi \eta+D \eta^{2}, \quad A \neq 0,
$$

and again obtain a series for the transformed streamfunction,

$$
\widetilde{\psi}=\eta \sum_{i, j=0}^{\infty} \tilde{a}_{i j+1} \xi^{i} \eta^{j} .
$$

For the degenerate case $\bar{\varepsilon}=0$, Eq. (23), we removed two fourth-order terms by an appropriate quadratic transformation. This can also be obtained here by suitable choices of $C$ and $D$. We find, when $\bar{\varepsilon}=0$,

$$
\tilde{a}_{04}=a_{04}+3 D a_{03} \text {, }
$$

and since

$$
\frac{\partial \widetilde{a}_{04}}{\partial D}=3 a_{03} \neq 0,
$$

it follows from the implicit function theorem that there exists a function $D=D(\bar{\varepsilon})$ with $D(0)=-a_{04} /\left(3 a_{03}\right)$ which solves $\tilde{a}_{04}=0$. By similar arguments, we can choose $C$ such that $\tilde{a}_{13}=0$. Furthermore,

$$
\tilde{a}_{21}=\frac{1}{2} A^{2} \partial_{x}^{2} \partial_{y} \psi\left(x_{0}, 0\right)+A C \partial_{x} \partial_{y} \psi\left(x_{0}, 0\right)
$$

We can solve $\tilde{a}_{21}=0$ for $x_{0}$ by noting that, when $x_{0}=0$, $\tilde{a}_{21}=0$ and

$$
\frac{\partial \widetilde{a}_{21}}{\partial x_{0}}=3 A^{2} a_{31} \neq 0 .
$$

The coefficient $B$ appears linearly in $\tilde{a}_{22}$, and hence we can obtain $\tilde{a}_{22}=0$ by solving for $B$. We omit the details.

With these simplifications we have

$$
\widetilde{\psi}=\eta\left(\widetilde{a}_{01}+\widetilde{a}_{02} \eta+\widetilde{a}_{11} \xi+\widetilde{a}_{12} \xi \eta+\widetilde{a}_{03} \eta^{2}+\widetilde{a}_{31} \xi^{3}\right)+\mathcal{O}_{5},
$$

where four coefficients are small,

$$
\begin{aligned}
\tilde{a}_{01}= & \partial_{y} \psi\left(x_{0}, 0\right), \\
\tilde{a}_{02}= & \left(D+\frac{1}{2 \lambda_{a}\left(x_{0}\right)}\right) \partial_{y} \psi\left(x_{0}, 0\right)+B \partial_{x} \partial_{y} \psi\left(x_{0}, 0\right), \\
\tilde{a}_{11}= & C \partial_{y} \psi\left(x_{0}, 0\right)+\partial_{x} \partial_{y} \psi\left(x_{0}, 0\right), \\
\tilde{a}_{12}= & \left(\frac{C}{\lambda_{a}\left(x_{0}\right)}-\frac{\lambda_{a}^{\prime}\left(x_{0}\right)}{\lambda_{a}\left(x_{0}\right)^{2}}\right) \partial_{y} \psi\left(x_{0}, 0\right) \\
& +\left(D-B C+\frac{1}{\lambda_{a}\left(x_{0}\right)}\right) \partial_{x} \partial_{y} \psi\left(x_{0}, 0\right) .
\end{aligned}
$$

Note that $\tilde{a}_{02}$ and $\tilde{a}_{12}$ can be expressed as linear combinations of the two other coefficients $\tilde{a}_{01}$ and $\tilde{a}_{11}$. Hence the streamfunction now only depends on two small parameters to fourth order. The codimension is two.

The remaining coefficients are nonzero:

$$
\tilde{a}_{03}=a_{03}+\mathcal{O}(\bar{\varepsilon}) \text {, }
$$

$$
\tilde{a}_{31}=A^{3} a_{31}+\mathcal{O}(\bar{\varepsilon}) .
$$

Choosing

$$
A=\sqrt[3]{\frac{a_{03}}{a_{31}}}+\mathcal{O}(\bar{\varepsilon})
$$

makes the coefficients to $\eta^{3}$ and $\xi^{3} \eta$ identical, and dividing by their common value we obtain

$$
\begin{aligned}
\widetilde{\psi}= & \eta\left[F+G \xi+(\alpha F+\beta G) \eta+(\gamma F+\delta G) \xi \eta+\eta^{2}+\xi^{3}\right] \\
& +\mathcal{O}_{5},
\end{aligned}
$$

where $F, G$ are small parameters. The four constants $\alpha, \beta, \gamma, \delta$ link the four small coefficients, as described in Eqs. (51). As the following analysis will show, the bifurcation structure does not depend on their value. Finally, we rename the coordinates back to $x, y$ and drop the $\mathcal{O}_{5}$ term to obtain the normal form

$$
\psi=y\left[F+G x+(\alpha F+\beta G) y+(\gamma F+\delta G) x y+y^{2}+x^{3}\right] .
$$

Critical points on the surface are found from

$$
u(x, 0)=\frac{\partial \psi}{\partial y}(x, 0)=F+G x+x^{3}=0,
$$

which has up to three solutions. The number of solutions changes when the discriminant of the cubic is zero, that is, when

$$
G=-3 \sqrt[3]{\frac{1}{4} F^{2}} .
$$

In the $F, G$ parameter plane, this is a cusp-shaped curve. One easily finds that the bifurcations on one branch of the cusp are bubble creation, and the bifurcations on the other branch are bubble merging. To find off-surface critical points, we consider $u(x, y)=0$ and $v(x, y) / y=0$. In these two equations, $F, G$ appear linearly, and we can solve to obtain

$$
F=-3 y^{2}+\mathcal{O}_{3}, \quad G=-3 x^{2}+\mathcal{O}_{3} .
$$

Bifurcation occurs when the Jacobian matrix $J$ of the vector field at the critical point has a zero determinant. Inserting (58), one finds

$$
\operatorname{det} J=36 x y^{2}+\mathcal{O}_{4} .
$$

Hence, to leading order, bifurcations off the surface occur for $x=0$, and, according to (58), for $G=0, F<0$. The bifurcations here are cusp bifurcations, where a center and a saddle merge and disappear. ${ }^{18}$ The bifurcation diagram is shown in Fig. 4.

\section{BIFURCATION OF PATTERNS FOR STOKES FLOW INSIDE A CIRCLE}

\section{A. Solution procedure}

As an application of the previous results we shall solve a simple two-dimensional incompressible Stokes flow inside the unit circle driven by rotlets. 


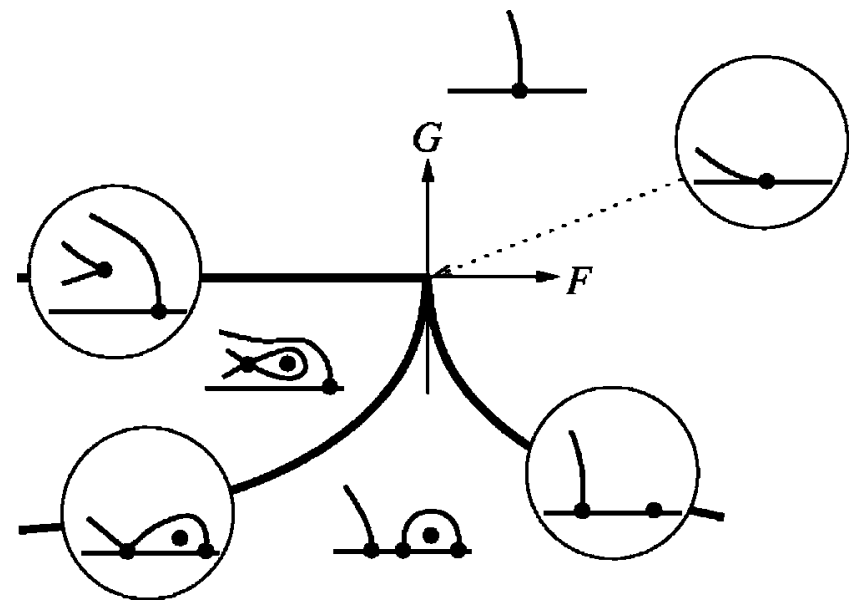

FIG. 4. Bifurcation diagram for the codimension two normal form (55). In each sector, bounded by the heavy bifurcation curves, the corresponding streamline pattern is shown. The degenerate patterns occurring on the bifurcation curves is shown inside circles. The bifurcation at $G=0, F<0$ is a creation/destruction of two off-surface critical points (cusp bifurcation). At $G=-3 \sqrt[3]{F^{2} / 4}, F<0$, a bubble merging bifurcation occurs; cf. the top row of Fig. 3. At $G=-3 \sqrt[3]{F^{2} / 4}, F>0$ a bubble creation bifurcation occurs; cf. the bottom row of Fig. 3 .

We use polar coordinates $(r, \theta)$ such that the velocity field is given by

$$
v_{r}=-\frac{1}{r} \partial_{\theta} \psi, \quad v_{\theta}=\partial_{r} \psi
$$

The streamfunction satisfies the biharmonic equation

$$
\Delta^{2} \psi(r, \theta)=0,
$$

for $0<r<1$ and $0 \leqslant \theta<2 \pi$. The no-flux boundary condition is

$$
\psi(1, \theta)=0,
$$

and, as $\partial_{n}=-\partial_{r}$ and $v_{\|}=v_{\theta}$, the Navier boundary condition (5) becomes

$$
\partial_{r} \psi(1, \theta)=-\lambda_{a} \partial_{r}^{2} \psi(1, \theta) .
$$

The radius of curvature $R=+1$ is constant and positive, since the flow domain is convex. Hence, $\lambda_{a}=\left(1 / \lambda_{0}-1\right)^{-1}$ is a constant.

We consider the case where the flow is driven by a point rotlet of unit strength centered at $(r, \theta)=(c, 0), c<1$. The rotlet streamfunction is $\psi_{1}=\log \rho$, where $\rho$ denotes the distance to the rotlet center. Following Ref. 12, we expand the streamfunction for $r<c$ and any $\theta$,

$$
\begin{aligned}
\psi_{1}(r, \theta)=\log \rho & =\log \sqrt{r^{2}+c^{2}-2 r c \cos \theta} \\
& =\left(\log r-\sum_{n=1}^{\infty} \frac{1}{n} \frac{c^{n}}{r^{n}} \cos n \theta\right) .
\end{aligned}
$$

The full solution to (61) is written as a sum of the rotlet streamfunction and a correction due to the boundaries

$$
\psi(r, \theta)=\psi_{1}(r, \theta)+\phi(r, \theta) .
$$

Assuming analyticity, the correction term can be expanded,

$$
\phi(r, \theta)=\sum_{n=0}^{\infty}\left(a_{n} r^{n}+b_{n} r^{n+2}\right) \cos n \theta,
$$

and inserting in the boundary conditions (63), the coefficients become

$$
\begin{aligned}
& a_{0}=\frac{1-\lambda_{a}}{2\left(1+\lambda_{a}\right)}, \quad b_{0}=-a_{0}, \\
& a_{n}=\frac{c^{n}}{n} \frac{\left(1+\lambda_{a}\right)(1+n)}{1+\lambda_{a}(1+2 n)}, \quad b_{n}=c^{n} \frac{\lambda_{a}-1}{1+\lambda_{a}(1+2 n)}, \\
& \text { for } n=1,2, \ldots .
\end{aligned}
$$

The solution procedure is immediately generalized by the superposition to flows generated by any number of rotlets of arbitrary strength.

This problem is also considered in Ref. 15, but with the erroneous boundary condition

$$
\partial_{r} \psi(1, \theta)=\lambda_{a} \partial_{r}^{2} \psi(1, \theta) .
$$

This is the relevant Navier boundary condition for the flow outside the unit disk, where the normal derivative at the boundary is $\partial_{n}=\partial_{r}$, and is correctly used for that case in Ref. 19.

\section{B. Numerical results}

Using the procedure described above, we have found streamline patterns for the flow generated by two rotlets. One rotlet of unit strength is held fixed at $(r, \theta)=(1 / 2, \pi)$. The other rotlet has strength $\sigma$ and is placed at $(r, \theta)=(c, \beta)$. The flow pattern thus depends on four parameters, including the slip length $\lambda_{a}$.

The series (66) converges slowly when $c$ is close to 1 . To avoid numerical problems, we restrict $c$ to [0;0.9]. The Taylor series for the streamfunction is truncated after 30 terms, where we obtain the $L_{2}$ norm of $\psi$ at the boundary to be less than $10^{-15}$.

To explore the parameter space, we have used the following scheme: For fixed values of $\sigma$ and $\lambda_{a}$ we scan the $(c, \beta)$ parameter space, counting the number of sign changes of the tangential velocity $v_{\theta}(1, \theta)$ at the boundary for each value of $(c, \beta)$. This gives an indication of the number of separation bubbles in the flow, as each zero of $v_{\theta}$ corresponds to a critical point on the surface.

Typical results are shown in Fig. 5, where the number of separation bubbles is indicated by a shade of gray. The boundaries between regions of different shades are curves of creation or merging bifurcations. Representative streamline patterns are shown in Fig. 6. On the large scale, it may appear that the dividing streamlines do not meet the surface at right angles, as predicted by the theory of Sec. III A. However, zooming to a scale comparable to the slip length $\lambda_{a}$, Fig. 6(d), we see that this qualitative feature of the streamline pattern is indeed fulfilled.

When $\sigma>0$ and the two rotlets have the same sense of rotation, only creation bifurcations are found; see Fig. 5(a) and 5(c). For $\sigma<0$, merging bifurcations also appear. In a 
(a)

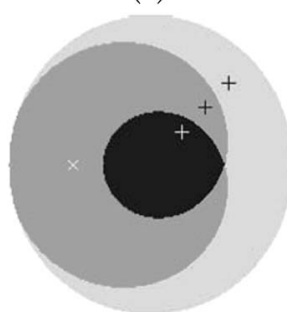

(c)

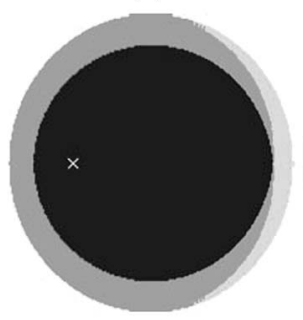

0 bubbles (b)

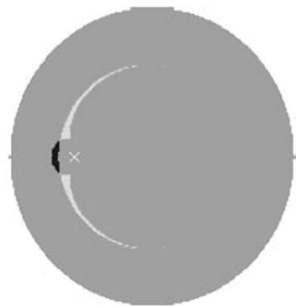

(d)

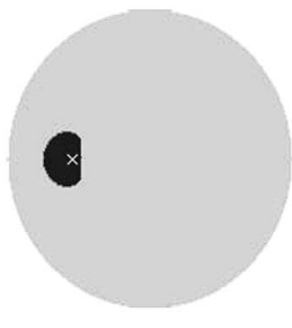

2 bubbles

FIG. 5. Bifurcation diagrams in the $(c, \beta)$ parameter plane for fixed values of $\sigma$ and $\lambda_{a}$. The fixed rotlet is marked by a $\times$. (a) $\sigma=0.5, \lambda_{a}=0.01$. Streamline patterns corresponding to the three markers + are shown in Fig. 6. (b) $\sigma=-0.5, \lambda_{a}=0.01$. (c) $\sigma=0.5, \lambda_{a}=0.2$. (d) $\sigma=-0.5, \lambda_{a}=0.2$.

blowup of Fig. 5(b), as shown in Fig. 7(d), we see a creation and a merging bifurcation curve meet in a codimension two point. In agreement with the theory of Sec. III C, the two curves meet in a cusp. Furthermore, the theory predicts the existence of a curve of off-surface bifurcations emanating from the codimension two point. To find bifurcation of offsurface critical points, we consider the two curves (isoclines) in the physical space where $v_{r}=0$ and $v_{\theta}=0$. Critical points are located at the intersection of the two isoclines, and by monitoring whether they intersect or not we have found the curve of off-surface bifurcations, as shown in Fig. 7(d).

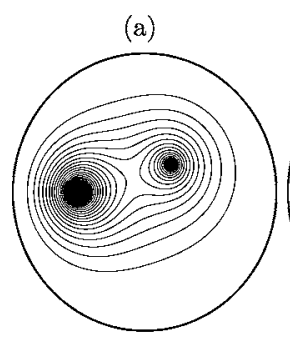

(c)

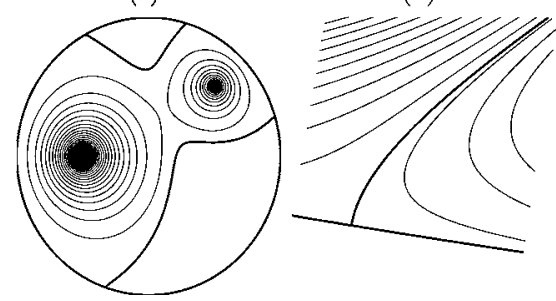

FIG. 6. Streamline patterns corresponding to the three markers in Fig. 5(a). In all cases $\beta=\pi / 4$. (a) $c=0.28$. (b) $c=0.50$. (c) $c=0.71$. The heavy lines are dividing streamlines from the on-wall stagnation points. (d) A blowup of the region close to the left on-wall stagnation point in (b).

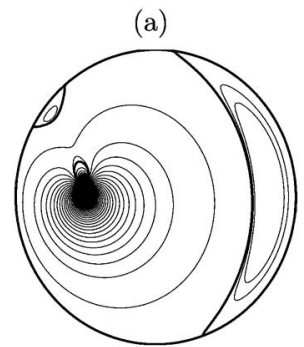

(c)

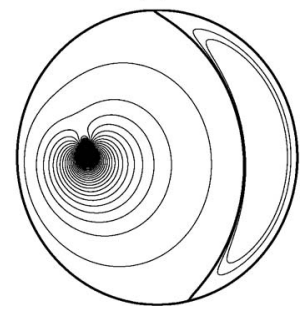

(b)

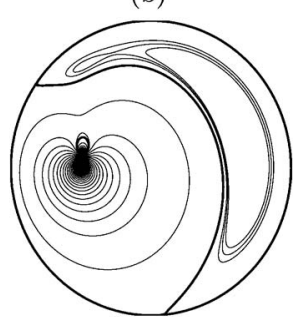

(d)

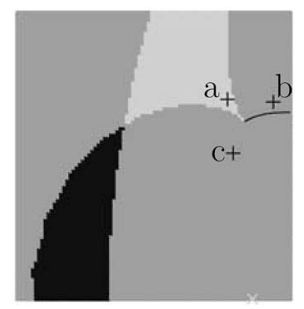

FIG. 7. Flow patterns close to a codimension 2 point, $(c, \beta)=(0.516,2.93)$ for $\sigma=-0.5, \lambda_{a}=0.01$, are shown in (a)-(c). A blowup of the corresponding bifurcation diagram in Fig. 5(b) is shown in (d). (a) $c=0.535, \beta=2.91$. (b) $c=0.502, \beta=2.91$. (c) $c=0.522, \beta=2.97$.

\section{CONCLUSIONS}

For 2D flows near a surface with a Navier slip boundary condition with a finite slip length, we have analytically obtained bifurcation diagrams for streamline patterns under the variation of one or two external parameters. The qualitative structure of the bifurcations and the possible streamline patterns do not, perhaps surprisingly, depend on the value of the slip length, nor on the curvature of the surface.

Furthermore, the two bifurcation diagrams, Figs. 3 and 4 , are, from a strict topological point of view, identical to the ones obtained for a no-slip surface, ${ }^{8}$ although the normal forms and their derivations are different. We find only a few qualitative differences between the streamline topology in the no-slip and the slip case that are of a geometric rather than a topological nature: For a regular critical point, the dividing streamline is orthogonal to the surface in the slip case. For no-slip flows, the angle can take any value, depending on the gradients of surface stress and pressure. For a degenerate critical point of order 3 , dividing streamlines are symmetric in the slip case. This does not necessarily occur in the no-slip case.

We have used the bifurcation theory to analyze the patterns in a simple Stokes flow generated by singularities. We have found both kind of bifurcations described theoretically. Bifurcations of critical points at the surface are easily found from the solution of the Stokes problem. Having located a bifurcation point of codimension two, the theory predicts further bifurcations off the surface that we subsequently have identified. Hence, the theory not only gives a basic overview of possible changes of streamline patterns, it also provides a guideline for a practical determination of bifurcations.

The present analysis may be extended to 3D flows. A bifurcation analysis is outside the scope of the present paper, but a basic fact is worth mentioning. Consider the no-slip 
flow close to a planar surface given as the $x, y$ plane in an $x y z$ coordinate system. It is well known ${ }^{20-22}$ that if the origin is a regular critical point, the streamlines are locally described as solutions to a linearized system of the form

$$
\left(\begin{array}{c}
\dot{x} \\
\dot{y} \\
\dot{z}
\end{array}\right)=\left(\begin{array}{ccc}
a_{11} & a_{12} & a_{13} \\
a_{21} & a_{22} & a_{23} \\
0 & 0 & -\frac{a_{11}+a_{22}}{2}
\end{array}\right)\left(\begin{array}{l}
x \\
y \\
z
\end{array}\right) .
$$

For a flow fulfilling the Navier boundary conditions (4) and (6), it is not difficult to show that the corresponding equations become

$$
\left(\begin{array}{c}
\dot{x} \\
\dot{y} \\
\dot{z}
\end{array}\right)=\left(\begin{array}{ccc}
a_{11} & a_{12} & 0 \\
a_{21} & a_{22} & 0 \\
0 & 0 & -\left(a_{11}+a_{22}\right)
\end{array}\right)\left(\begin{array}{l}
x \\
y \\
z
\end{array}\right) .
$$

In the latter case, a streamline going into the fluid from the critical point will be orthogonal to the surface. This need not be the case for Eqs. (70), similar to the 2D flow. Whether the bifurcation structure, depending on nonlinear terms of the velocity field, differs in the two cases, remains to be seen.

${ }^{1}$ E. Lauga, M. P. Brenner, and H. A. Stone, "Microfluidics: The no-slip boundary condition," in Handbook of Experimental Fluid Dynamics, edited by J. Foss, C. Tropea, and A. Yarin (Springer-Verlag, New York, to be published), Chap. 15.

${ }^{2}$ D. C. Tretheway and C. D. Meinhart, "Apparent fluid slip at hydrophobic microchannel walls," Phys. Fluids 14, L9 (2002).

${ }^{3}$ C.-H. Choi, J. A. Westin, and K. S. Breuer, "Apparent slip flows in hydrophilic and hydrophobic microchannels," Phys. Fluids 15, 2897 (2003).

${ }^{4}$ E. Lauga and H. A. Stone, "Effective slip in pressure-driven Stokes flow," J. Fluid Mech. 489, 55 (2003).

${ }^{5}$ S. Gogte, P. Vorobieff, R. Truesdell, A. Mammoli, F. van Swol, P. Shah, and C. J. Brinker, "Effective slip on textured superhydrophobic surfaces," Phys. Fluids 17, 051701 (2005).

${ }^{6}$ C. Y. Wang, "Flow over a surface with parallel grooves," Phys. Fluids 15, $1114(2003)$

${ }^{7}$ P. G. Bakker, Bifurcations in Flow Patterns (Klüwer Academic, Dordrecht, 1991).

${ }^{8}$ J. N. Hartnack, "Streamline topologies near a fixed wall using normal forms," Acta Mech. 136, 55 (1999).

${ }^{9} \mathrm{M}$. Brøns, "Streamline topology—Patterns in fluid flows and their bifurcations," Adv. Appl. Mech. 41, 38 (2006).

${ }^{10}$ H. Aref, "Stirring by chaotic convection," J. Fluid Mech. 143, 1 (1984).

${ }^{11}$ S. C. Jana, G. Metcalfe, and J. M. Ottino, "Experimental and computational studies of mixing in complex Stokes flow: The vortex mixing flow and multicellular cavity flows," J. Fluid Mech. 269, 19 (1994).

${ }^{12} \mathrm{~V}$. V. Meleshko and H. Aref, "A blinking rotlet model for chaotic advection," Phys. Fluids 8, 3215 (1996).

${ }^{13}$ P. Daripa and D. Palaniappan, "Singularity induced exterior and interior Stokes flows," Phys. Fluids 13, 3134 (2001).

${ }^{14}$ T. J. Price, T. Mullin, and J. J. Kobine, "Numerical and experimental characterization of a family of two-roll-mill flows," Proc. R. Soc. London, Ser. A 459, 117 (2003).

${ }^{15}$ D. Palaniappan and P. Daripa, "Interior Stokes flow with stick-slip boundary conditions," Physica A 297, 37 (2001).

${ }^{16}$ D. Einzel, P. Panzer, and M. Liu, "Boundary condition for fluid flow: Curved or rough surfaces," Phys. Rev. Lett. 64, 2269 (1990).

${ }^{17}$ S. Wiggins, Introduction to Applied Nonlinear Dynamical Systems and Chaos (Springer-Verlag, New York, 1990).

${ }^{18} \mathrm{M}$. Brøns and J. N. Hartnack, "Streamline topologies near simple degenerate critical points in two-dimensional flow away from boundaries," Phys. Fluids 11, 314 (1999).

${ }^{19}$ D. Palaniappan and P. Daripa, "Exterior Stokes flow with stick-slip boundary conditions," Z. Angew. Math. Phys. 53, 281 (2002).

${ }^{20}$ M. Tobak and D. J. Peake, "Topology of three-dimensional separated flows," Annu. Rev. Fluid Mech. 14, 61 (1982).

${ }^{21}$ M. S. Chong, A. E. Perry, and B. J. Cantwell, "A general classification of three-dimensional flow fields," Phys. Fluids A 2, 765 (1990).

${ }^{22}$ J. N. Hartnack, "Structural changes in incompressible flow patterns," Ph.D. thesis, Department of Mathematics, Technical University of Denmark, 1999. 